\title{
Sputum and plasma adiponectin levels in clinically stable adult cystic fibrosis patients with CFTR I1234V mutation
}

Atqah AbdulWahab ( $\square$ atqah2015@gmail.com )

Sidra Medical and Research Center

Mona Allangawi

Hamad Medical Corporation

Merlin Thomas

Hamad Medical Corporation

Ilham Bettahi

Hamad Medical Corporation

Siveen K. Sivaraman

Hamad Medical Corporation

Jayakumar Jerobin

Hamad Medical Corporation

Prem Chandra

Hamad Medical Corporation

Abdul-Badi Abou-Samra

Hamad Medical Corporation

Manjunath Ramanjaneya

Hamad Medical Corporation

\section{Research}

Keywords: cystic fibrosis, CFTR I1234V, adiponectin, adults

Posted Date: January 16th, 2020

DOI: https://doi.org/10.21203/rs.2.19105/v2

License: (a) (1) This work is licensed under a Creative Commons Attribution 4.0 International License.

Read Full License

Version of Record: A version of this preprint was published at Translational Medicine Communications on February 13th, 2020. See the published version at https://doi.org/10.1186/s41231-020-00053-2. 


\section{Abstract}

Background: Cystic fibrosis (CF) lung disease is associated with chronic inflammation leading to progress in lung function. Adiponectin is a predominantly anti-inflammatory adipokine that may have a role in CF lung. This study aims to determine total sputum and total plasma adiponectin levels in clinically stable adults CF patients with CFTR I1234V mutation, compared to plasma adiponectin levels in healthy controls and to investigate their correlations with body mass index (BMI) and spirometry in patients with CF. Methods: A cross-sectional study comprises $17 \mathrm{CF}$ patients and 18 healthy controls. Adiponectin levels were measured by magnetic bead-based multiplex assay. Results: The mean age of adult CF patients was 22.9 years \pm 3.8 (18-30) and $76.5 \%$ CF patients had pancreatic sufficiency. The mean BMI in healthy controls was slightly higher than CF patients. The mean sputum adiponectin level was significantly lower than plasma adiponectin levels in CF patients and healthy controls $(p<0.001)$, whereas no significant difference in plasma adiponectin levels between CF patients and healthy controls. The mean sputum adiponectin level was observed to be higher in CF patients with pancreatic insufficiency. Sputum adiponectin level was correlated positively with plasma adiponectin level in CF patients $(r=0.47, p=0.06)$. Sputum and plasma adiponectin levels in CF patients were correlated negatively with $\mathrm{BMI}$ and percentage predicted forced expiratory volume in 1 second (FEV1) and Forced vital capacity (FVC). Conclusions Sputum adiponectin may provide a minimally invasive tool in the assessment of an inflammatory status in CF patients. Further larger study to address any difference in sputum and plasma adiponectin levels among CF patients with pancreatic sufficiency versus pancreatic insufficiency.

\section{Background}

Cystic fibrosis (CF) is the most common life-shortening genetic disease caused by a mutation in the cystic fibrosis transmembrane conductance regulator (CFTR) gene leading to altered chloride ion exchange and mucus hypersecretion in the affected organs [1]. To date more than 2200 mutations have been identified in the CFTR gene and all these types of mutations lead to a defective or absence of CFTR protein [2]. CFTR I1234V mutation is one of the most common mutations among Arabs in the state of Qatar and is usually associated with pancreatic sufficiency [3-5].

CF-associated lung disease is characterized by multiple alterations including hyper viscous mucus, and chronic neutrophilic inflammation with recurrent bacterial infections, mainly caused by Pseudomonas aeruginosa and Staphylococcus aureus and associated with reduced lung function [6-11].

Adiponectin is predominantly produced by adipocytes and belongs to the adipokine family. It was believed that adiponectin was only expressed by adipocytes, however recent research indicates that it may be expressed in the lung as well [12]. Adiponectin is mainly anti-inflammatory role that involves inhibition of pro-inflammatory mediators such as tumor necrosis factor-alpha (TNF-a) and interleukin (IL6) and promotion of anti-inflammatory mediators (IL- 10 and IL -1 receptor antagonist) that play a part in the development of exacerbation or control of inflammation [13-14]. Furthermore, adiponectin may 
account in a number of metabolic processes, including glucose and fatty acid metabolism where its levels reduced with increasing obesity, type 2 diabetes, the and cardiovascular disease compared to healthy controls matched by BMI [15-17]. Serum adiponectin circulates as complexes of 3 different molecular weights ((low-molecular weight (LMW) (trimers); medium-molecular weight (MMW) (hexamers); and high-molecular-weight (HMW or higher-order multimers)). Its properties are mediated by specific receptors that are widely expressed with AdipoR $\mathrm{R}_{1}, \mathrm{AdipoR}_{2}$, and T-cadherin being present on epithelial and endothelial pulmonary cells, expressing potentially a vital role in lung physiology [18-19].

Airway inflammation is a hallmark feature of CF lung disease and has an impact on the quality of life and survival. Therefore, it is important to focus on investigating relevant pulmonary biomarkers to evaluate airway inflammation in CF. Systemic (blood-based) markers of inflammation are ideal as blood measurements are easily standardized, reproducible, and can be obtained from subjects of any age and disease severity. An important question, however, remains to be answered if any relationship between plasma and sputum adiponectin levels. The main objective of the study is to determine total sputum and total plasma adiponectin levels in clinically stable adult CF patients with CFTR I1234V mutation, compared to plasma adiponectin levels in healthy control. The secondary objective is to investigate whether the sputum or systemic levels of adiponectin have correlations with BMI and spirometry in patients with $\mathrm{CF}$.

\section{Methods}

\section{Study design and participants}

In this prospective cross-sectional study, CF patients enrolled in the current study were clinically stable CF adults with CFTR I1234V mutation attending adult CF clinics as routine care at Hamad Medical Corporation (HMC), Doha, Qatar. The diagnosis of CF was made on one or more phenotypic features consistent with $\mathrm{CF}$, positive family history of $\mathrm{CF}$ in siblings and close relatives, confirmed by elevated the concentration of sweat chloride (>60 mmol/l) on two different occasions and CF genetic testing.

Inclusion criteria included all CF patients $\geq 18$ years old, who were able to expectorate sputum and free of active infections for more than 4 weeks prior to study enrollment and no acute chest exacerbation among CF patients based on recent definition [20].

Exclusion criteria included all CF patients who were smoking within the last two months; pregnancy, nursing mothers and CF patients who underwent a lung transplant. Control subjects were recruited from volunteers visiting the hospital with patients who were non-smoker and free from acute infection during the 4 weeks preceding the study.

\section{Study measures and data variables}

Bodyweight was recorded using an electronic platform scale and standing height measurement using a stadiometer. BMI was computed by dividing weight in $\mathrm{kg}$ by the height squared in meters. Clinical data 
were obtained from CF subjects; sputum was collected for microbiology per CF consensus guidelines [21]. All CF subjects perform spirometry tests in the respiratory laboratory

unit in accordance with the guidelines $f$ the American Thoracic Society [22]. The best recorded forced expiratory volume in 1 second (FEV1) using Spiro bank, MIR, Italy. The maximum of three appropriate measurements is recorded with $<15 \%$ variation. Forced vital capacity (FVC; in liters), forced expiratory volume in 1second (FEV1; in liters,), FEV1/FVC (in \%) are measured and expressed as a percent of predicted normal using standard equations [22].

Pancreatic insufficiency was defined as those with low fecal elastase level and required daily pancreatic enzyme supplementation with meals and snacks.

Measurement of adiponectin in plasma and sputum samples: The plasma and sputum were collected from each CF patient while for the control subjects only plasma was collected for adiponectin measurement. Plasma and sputum adiponectin levels were measured using Bio-Plex Pro Human Diabetes Adiponectin Assay (Cat \# 171a7003m). All samples were assayed in duplicate. The lower limit of detection with the adiponectin assay was $171 \mathrm{pg} / \mathrm{ml}$. The Intra-assay coefficients of variation were $4 \%$ and inter-assay coefficients of variation was $2 \%$.

\section{Statistical Analysis}

Data are presented as mean \pm standard deviation (SD) or median (quartile range) for data with a skewed distribution. Categorical data values were expressed as frequencies (percentages). Differences in their mean values between CF patients and healthy controls were compared using unpaired Student's t-test and Mann-Whitney $U$ test for skewed data distribution. Associations between two or more categorical variables (gender, CF patients and healthy controls) were examined using Chi-square $(\chi 2)$ test or Fisher Exact test as appropriate.

Pearson's correlation was applied to assess the strength of the linear relationship between two or more quantitative variables (to evaluated baseline concentrations of sputum and serum adiponectin, in the of CF patients and assess the relationship to FEV1 \%, FVC \%, FEV1/FVC and BMI). Key findings presented using appropriate statistical graphs (scatter and Box plots). All $P$ values presented were two-sided, and $P$ values $<0.05$ was considered as statistically significant. All Statistical analyses were done using statistical packages SPSS 23.0 (SPSS Inc. Chicago, IL) and Epi-info (Centers for Disease Control and Prevention, Atlanta, GA) software.

\section{Results}

The characteristics of $17 \mathrm{CF}$ adults patients and 18 healthy control subjects are presented in Table1.The mean age of adult CF patients was $22.9 \pm 3.8$ years (18-30) and 13/17 (76.47\%) CF patients had pancreatic sufficiency, one had CF-related diabetes mellitus (CF-DM) and none of CF patients had CFrelated liver disease. Thirteen were colonized with Pseudomonas aeruginosa and one patient had 
methicillin resistant staphylococcus (MRSA). The differences in the mean BMI between CF patients $(23.82 \pm 5.22)$ and healthy control subjects $(25.33 \pm 3.46)$ found to be statistically insignificant $(p>0.05)$. However, the mean BMI in CF patients with pancreatic sufficiency $(25.3 \pm 4.9)$ was significantly higher than CF with pancreatic insufficiency $19.25 \pm 3.3(p=0.038)$.

The mean total plasma adiponectin levels between CF patients and healthy controls $(10.71 \pm 7.63 \mu \mathrm{g} / \mathrm{ml}$ \& $9.11 \pm 6.19 \mu \mathrm{g} / \mathrm{ml}$ respectively) did not differ significantly; median plasma adiponectin level in CF patients and healthy control were 8.54 (range 1.66 to 31.28 )) and 7.63 (range 1.70 to 23.52 ) respectively. The mean sputum adiponectin levels in CF patients was $0.91 \pm 0.79$, median $0.85(0.01-2.42)$ and significantly lower to plasma adiponectin levels in CF patients and healthy controls $(p<0.001)$ Fig1. Sputum adiponectin level was correlated positively with plasma adiponectin level in CF patients $(r=0.47$, $\mathrm{p}=0.06)$ Fig2. A negative correlation was found between age and total plasma adiponectin in CF patients, control subjects and total sputum adiponectin respectively $(r=-0.542, \mathrm{p}=0.024 ; r=-0.231, \mathrm{p}=0.372 ; r=$ $-0.385, p=0.127)$.

The mean sputum and plasma adiponectin level were higher in CF patients with pancreatic insufficiency $1.24 \pm 0.12,11.51 \pm 7.17$ respectively versus $C F$ patients with pancreatic sufficiency $0.80 \pm 0.89,10.47 \pm$ 8.03. Sputum and plasma adiponectin levels in CF patients were correlated negatively with $\mathrm{BMI}(r=-0.40$, $\mathrm{p}=0.12$ and; $r=-0.55, \mathrm{p}=0.03$ respectively) Fig3A, 3B. Spirometric parameters $\mathrm{FEV} 1 \%$ and $\mathrm{FVC} \%$ correlated positively with $\mathrm{BMI}(r=0.57, \mathrm{p}=0.022$ and; $r=0.56, \mathrm{p}=0.024$ respectively). There were weak inverse correlations between total serum and sputum adiponectin and FEV1 \%, FVC\% and no correlation with FEV1/ FVC \%.

\section{Discussion}

The lung is one of the important organs for adiponectin effects and consequently, adiponectin abnormalities may be related to lower airway disease such as asthma, CF and COPD [23-25].

Both airway infection and inflammation played a role in the deterioration of CF lung disease and accordingly assessment of inflammatory cells, cytokines, and anti-inflammatory molecules is essential for monitoring the progression of chronic CF lung disease. It has been reported that elevated sputum concentrations of neutrophils and inflammatory biomarkers to be clinically relevant in patients with acute chest exacerbation compared with those who were clinically stable [26-27].

We investigated whether stable adult CF patients with mild CFTR genotype (homozygous I1234V mutation) with pancreatic sufficiency modulated sputum and plasma adiponectin levels. The finding of this study demonstrated no significant differences in total plasma adiponectin concentrations in stable adult CF patients compared to healthy controls, although the mean BMI was found to be higher in healthy controls compared to CF patients, however their differences were statistically insignificant.

To our knowledge, we reported for the first time in a cohort of stable adult CF patients with homozygous CFTR I1234V mutation which demonstrated a positive correlation between plasma and sputum 
adiponectin. However, a mean sputum and plasma adiponectin levels were higher CF patients with pancreatic insufficiency compared to those CF patients with pancreatic sufficiency, which may suggest an augmented inflammatory process and possible disease severity more among CF patients with pancreatic insufficiency versus pancreatic sufficiency.

Adiponectin may have a potential role in the inflammatory process in lower airway diseases such as asthma, COPD and CF as well as critical illnesses such as respiratory failure with and without sepsis [28]. The present study demonstrated no difference in plasma adiponectin between stable adult CF patients and healthy controls. Recent studies have shown conflicting findings on the role of adiponectin in inflammatory lung conditions and only a few researches have been conducted on the role of circulating adiponectin in CF which was found to be inconsistent among different studies [29-31]. In one report, CF patients were found to have elevated adiponectin levels despite increased visceral adipose tissue mass, higher serum CRP levels, and similar levels of insulin resistance compared with a control population matched by BMI, age, and sex [29]. Another study reported by Hammana et al demonstrated normal adiponectin levels in the large cohort of CF patients despite abnormal glucose tolerance or diabetes and subclinical chronic inflammation [30]. Other study demonstrated lower adiponectin in CF children than in healthy children and higher resistin levels [31]. The discrepancy between studies can be explained by involved different age groups, ethnicity, variations, the severity of disease and organ involvement including pancreatic status liver involvement, nutritional status, and metabolic disorders.

FEV1\% is the currently used Spirometric predictor of morbidity and mortality in patients of CF. A recent study reported no association between adiponectin and lung function after adjusting for covariates related to adiposity in a population-based sample [32].

In contrast, the main findings of the present study demonstrated sputum and plasma adiponectin levels were inversely weakly correlated with Spirometric percentage predicted FEV1 and FVC suggesting that systemic and sputum inflammation might play a role in the development of airway obstruction. This is in agreement with other pulmonary diseases as in COPD subjects where demonstrated systemic adiponectin levels inversely associated with FEV1 [28]. In contrast to a recent study in children with CF observed no correlation between BMI and spirometry and adiponectin levels [31].

The results of our study should be considered as preliminary findings with some limitations. First, this study was designed to be cross-sectional that limits a causal link between sputum and plasma adiponectin levels and lung function changes. Other limitation includes a small number of adult CF patients with homozygous $11234 \mathrm{~V}$ mutation, the majority with pancreatic sufficiency and a varying degree of disease severity, making it a heterogeneous population in certain tribe.

\section{Conclusions}

Sputum adiponectin may provide a minimally invasive tool in the assessment of inflammatory markers in CF patients. Further studies are warranted to assess the possible role of adiponectin as an inflammatory 
biomarker in CF lung disease with pancreatic sufficiency versus pancreatic insufficiency in a larger CF cohort study

\section{Abbreviations}

CF: Cystic Fibrosis; CFTR: cystic fibrosis transmembrane conductance regulator; BMI: Body mass index; forced expiratory volume in 1 Second: FEV1; forced vital capacity: FVC. TNF-a : tumor necrosis factoralpha; IL-6: interleukin -6; LMW: low-molecular weight; MMW: medium-molecular weight; HMW: highmolecular weight; $\mathrm{CF}$ related diabetes mellitus: CF-DM; methicillin resistant staphylococcus: MRSA; chronic obstructive pulmonary disease: COPD.

\section{Declarations}

\section{Ethics approval and consent to participate}

This study was approved by the research and ethics committee (Institutional Review Board) of the Hamad Medical Corporation (RC 16226/16). The requirement of participant's informed and written consent was obtained from the IRB of Hamad Medical Corporation.

\section{Consent for publication}

Not applicable

\section{Availability of data and material}

Due to our local Institutional review board policy, we do not have permission to make the data sets on which the conclusions of the paper rely publicly available.

\section{Competing interests}

The authors declare that they have no competing interests. An abstract of this paper was presented at the Poster Session: Sputum and plasma adiponectin levels in clinically stable adult cystic fibrosis patients with CFTR I1234V mutation. In (European Respiratory Society) ERS Annual Congress Paris, 15-19 September 2018. The abstract was published in European Respiratory Journal 2018; Vol 52 Issue suppl 62

\section{Funding}

This research study was supported by the Medical Research Center, Hamad Medical Corporation (RC 16226/16) responsible for granting scientific and regulatory approval and budget provided covers laboratory investigations.

\section{Authors'contributions}


Atqah A: Conceptualized and designed the study, drafted the initial manuscript, reviewed and revised the manuscript, and approved the final manuscript as submitted. MA,MT: Designed the data collection and subjects recruitments. PC: carried out the statistical analyses, reviewed the manuscript. IB, SKS, and JJ: carried out the laboratory method, data collection and interpretation. AA: supervised laboratory work. MR: laboratory data method collection and contribute in writing the manuscript. All authors approved the final manuscript as submitted

\section{Acknowledgements}

We would like to thank Medical Research Center of the Hamad Medical Corporation for all their supports in this research study.

\section{References}

1. O'Sullivan, B.P. and P. Flume. The clinical approach to lung disease in patients with cystic fibrosis. Semin.Respir.Crit. Care. Med. 2009; 30:505-13.

2. Genomic Resource Centre. Genes and Human Disease. Available from: http://www. who.int/genomics/public/genetic diseases/en/index2. html.『

3. Abdul Wahab A, Al Thani G, Dawod ST, Kambouris M, Al Hamed M. Heterogeneity of the Cystic Fibrosis Phenotype in a large Kindred Family in Qatar With Cystic Fibrosis Mutation (11234V). J Trop Pediatr .2001;47:110-12.

4. Banjar, H., et al., Geographic distribution of cystic fibrosis transmembrane regulator gene mutations in Saudi Arabia. Ann Trop Paediatr .1999; 19:69-73.

5. Abdel Rahman H, Abdul Wahab A, Al Rahman MO, Mostafa OA. Faecal elastase -1 concentration in cystic fibrosis patients with CFTR I1234V mutation. Acta Paediatr .2006; 95:1066-69.

6. Sagel SD, Wagner BD, Anthony MM, Emmett P, Zemanick ET. Sputum biomarkers of inflammation and lung function decline in children with cystic fibrosis. Am JRespir Crit Care Med. 2012; 186:85765.

7. Cohen-Cymberknoh M, Kerem E, Ferkol T, Elizur A. Airway inflammation in cystic fibrosis: molecular mechanisms and clinical implications. Thorax. 2013; 68:1157-62.

8. Kreda SM, Davis CW, Rose MC. CFTR, mucins, and mucus obstruction in cystic fibrosis. Cold Spring Harb Perspect Med .2012;2: a009589.

9. Lobo J, Rojas-Balcazar JM, Noone PG. Recent advances in cystic fibrosis. Clin Chest Med .2012;33:307-28.

10. Lyczak, J.B., C.L. Cannon, and G.B. Pier. Lung infections associated with cystic fibrosis. Clin Microbiol Rev. 2002; 2: 194-222.

11. Elizur, A., C.L. Cannon, and T.W. Ferkol, Airway inflammation in cystic fibrosis. Chest. 2008; 2: 489-95.

12. Miller M, Cho JY, Pham A, Ramsdell J, Broide DH. Adiponectin and functional adiponectin receptor 1 are expressed by airway epithelial cells in chronic obstructive pulmonary disease. J Immunol 2009; 
$1: 684-91$.

13. Ali Assad, N. and A. Sood. Leptin, adiponectin and pulmonary diseases. Biochimie .2012; 10: 2180189.

14. Wolf, A.M., et al., Adiponectin induces the anti-inflammatory cytokines IL-10 and IL-1RA in human leukocytes. Biochem Biophys Res Commun. 2004;2: 630-35.

15. Lara-Castro C, Fu Y, Chung BH, Garvey WT. Adiponectin and the metabolic syndrome: mechanisms mediating risk for metabolic and cardiovascular disease. Curr Opin Lipidol.2007;18:263-70.

16. Galic S, Oakhill JS, Steinberg GR. Adipose tissue as an endocrine organ. Mol Cell Endocrinol. 2009;316:129-39.

17. Yamauchi T, Kadowaki T. Physiological and pathophysiological roles of adiponectin and adiponectin receptors in the integrated regulation of metabolic and cardiovascular diseases. Int $\mathrm{J}$ Obes (Lond). 2008;32(Suppl 7):S13-18.

18. Bianco A, Nigro E, Monaco ML, Matera MG, Scudiero O, Mazzarella G, et al. The burden of obesity in asthma and COPD: Role of adiponectin. Pulm Pharmacol Ther. 2017; 43:20-25.

19. Takemura Y, Ouchi N, Shibata R, Aprahamian T, Kirber MT, Summer RS, et al.Adiponectin modulates inflammatory reactions via calreticulinreceptor-dependent clearance of early apoptotic bodies. J Clin Invest 2007; 117:375-86.

20. Abbott J, Holt A, Hart A, Morton AM, MacDougall L, Pogson M, et al. What defines a pulmonary exacerbation? The perceptions of adults with cystic fibrosis. J Cyst Fibros. 2009; 8:356-59.

21. Huang YJ, LiPuma JJ. The Microbiome in Cystic Fibrosis. Clin Chest Med. 2016; 37:59-67.

22. Standardization of Spirometry, 1994 Update. American Thoracic Society. Am J Respir Crit Care Med. 1995; 152: 1107-36.

23. Eckrich J, Zissler UM, Serve F, Leutz P, Smaczny C, Schmitt-Grohé S, Fussbroich D, Schubert R, Zielen S, Eickmeier O. Airway inflammation in mild Cystic fibrosis. J Cyst Fibros. 2017; 16:107-15.

24. Montgomery ST, Dittrich AS, Garratt LW, Turkovic L, Frey DL, Stick SM, Mall MA, Kicic A; AREST CF. Interleukin-1 is associated with inflammation and structural lung disease in young children with cystic fibrosis. J Cyst Fibros. $2018 ; 17: 715-22$

25. McElvaney OJ, McElvaney NG. Targeting Interleukin-8 in Cystic Fibrosis: Enough Not Too Much. Am J Respir Cell Mol Biol. 2018; May 3

26. Lago F, Dieguez C, Gómez-Reino J, Gualillo O. Adipokines as emerging mediators of immune response and inflammation. Nat Clin Pract Rheumatol. 2007;3: 716-72.

27. Pavord ID, Pizzichini MM, Pizzichini E, Hargreave FE. The use of induced sputum to investigate airway inflammation. Thorax $.1997 ; 52,498-501$

28. Garcia P, Sood A. Adiponectin in pulmonary disease and critically ill Patients. Curr Med Chem. 2012; 19:5493-500. Review.

29. Moriconi N, Kraenzlin M, Müller B, Keller U, Nusbaumer CP, Stöhr S, Tamm M, Puder JJ. Body composition and adiponectin serum concentrations in adult patientswith cystic fibrosis. J Clin 
Endocrinol Metab 2006 ;91:1586-90.

30. Hammana I, Malet A, Costa M, Brochiero E, Berthiaume Y, Potvin S, Chiasson JL,Coderre L, RabasaLhoret R. Normal adiponectin levels despite abnormal glucosetolerance (or diabetes) and inflammation in adult patients with cystic fibrosis. Diabetes Metab. 2007;33:213-219.

31. Machura E, Szczepańska M, Świętochowska E, Halkiewicz F, Barć-Czarnecka M, Ziora K, Ziora D. Evaluation of adipokines in children with cystic fibrosis. Endokrynol Pol. 2018; 69:128-34

32. Caspersen NF, Røsjø H, Flyvbjerg A, Bjerre M, Randby A, Hrubos-Strøm H, Omland T, Einvik G. The association between circulating adiponectin levels, lung function and adiposity in subjects from the general population; data from the Akershus Sleep Apnea Project. BMC Pulm Med .2018; 18: 54.

\section{Table 1}

Table 1 Demographic and clinical data of CF and control subjects.

Data are expressed as mean \pm SD. $\square$ CF: cystic fibrosis, M: male, F: female, BMI: body mass index, CFRD: cystic fibrosis related diabetes, NS: nonsignificant.

\section{Figures}




\begin{tabular}{|c|c|c|c|}
\hline CF $(n=17)$ & $\begin{array}{l}\text { Control } \\
(\mathrm{n}=18)\end{array}$ & $\mathrm{p}$ & \\
\hline Gender (M/F) & $10 / 7$ & $9 / 9$ & - \\
\hline Age (years) & $22.94 \pm 3.78$ & $\begin{array}{l}33.56 \pm \\
11.35\end{array}$ & 0.0008 \\
\hline Pancreatic insufficiency & $\begin{array}{l}4 / 17 \\
(23.53 \%)\end{array}$ & $0 \%$ & - \\
\hline $\mathrm{BMI}(\mathrm{kg} / \mathrm{m} 2)(\operatorname{mean} \pm \mathrm{SD})$ & $23.82 \pm 5.22$ & $25.33 \pm 3.46$ & 0.318 \\
\hline CFRD & $\begin{array}{l}1 / 17 \\
(5.88 \%)\end{array}$ & $0 \%$ & - \\
\hline $\begin{array}{l}\text { Pseudomonas aeruginosa colonized in lower } \\
\text { respiratory secretion }\end{array}$ & $\begin{array}{l}13 / 17 \\
(76.5 \%)\end{array}$ & - & - \\
\hline$M R S A$ & $\begin{array}{l}1 / 17 \\
(5.88 \%)\end{array}$ & - & - \\
\hline \%FEV1 (mean \pm SD ) & $71.94 \pm 17.31$ & - & - \\
\hline$\%$ FVC $($ mean \pm SD $)$ & $77.65 \pm 14.65$ & - & - \\
\hline$\% F E V 1 / F V C($ mean \pm SD $)$ & $79.94 \pm 7.89$ & - & - \\
\hline
\end{tabular}




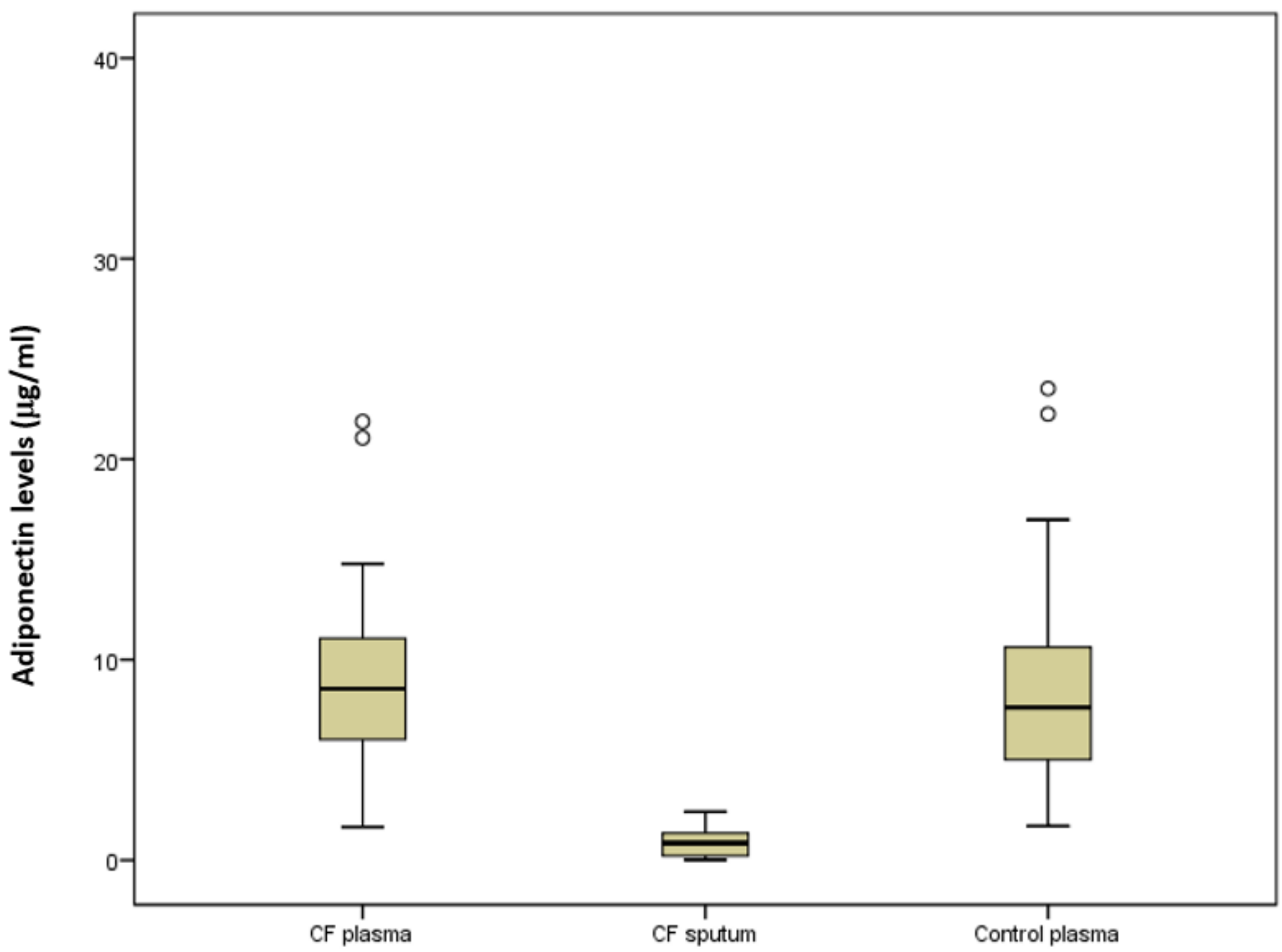

Figure 1

Distribution of adiponectin levels in 3 groups (serum adiponectin in CF, serum adiponectin in healthy control and sputum adiponectin in $\mathrm{CF}$ 


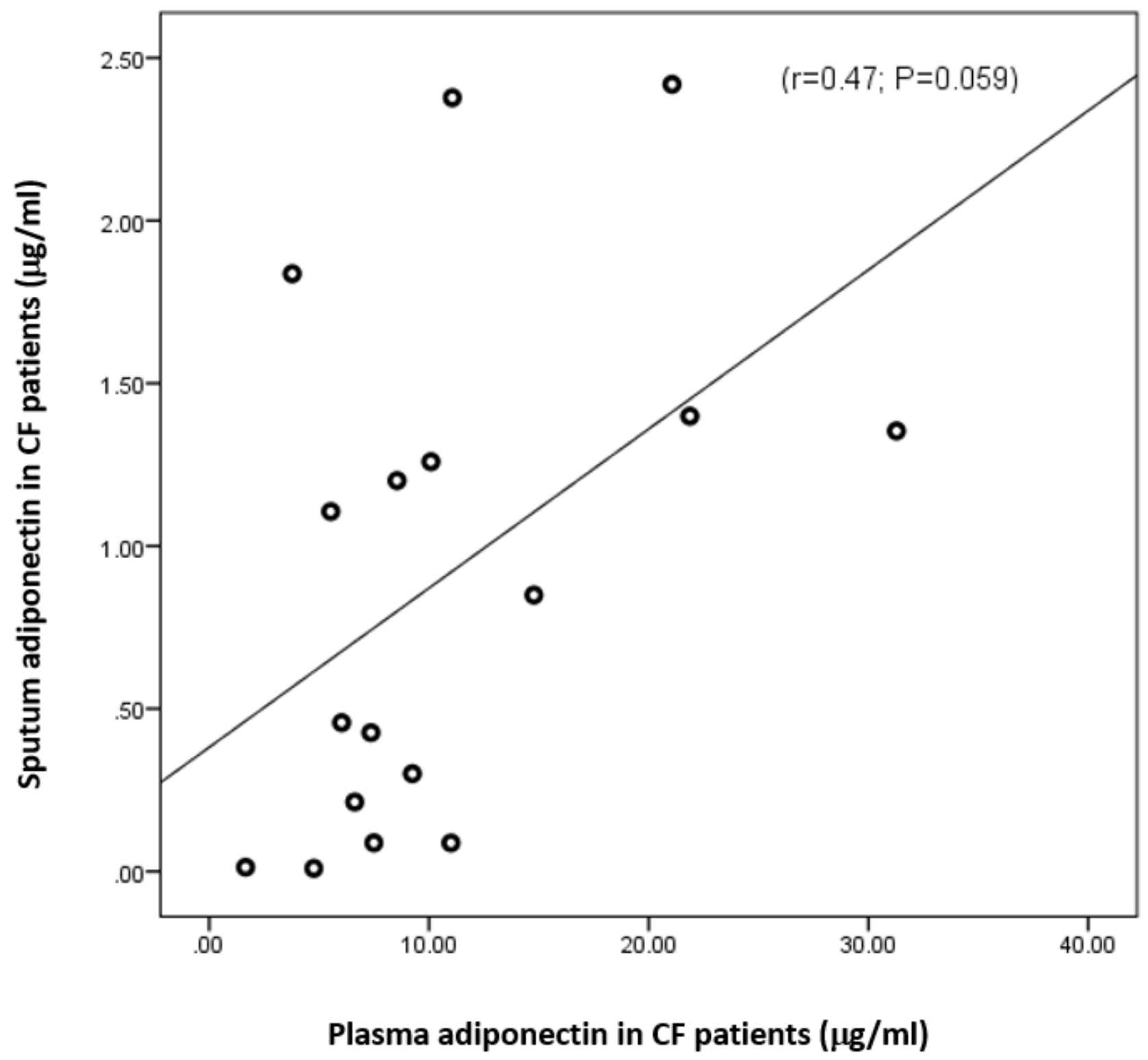

Figure 2

Correlation between sputum adiponectin level and plasma adiponectin level in CF patients 

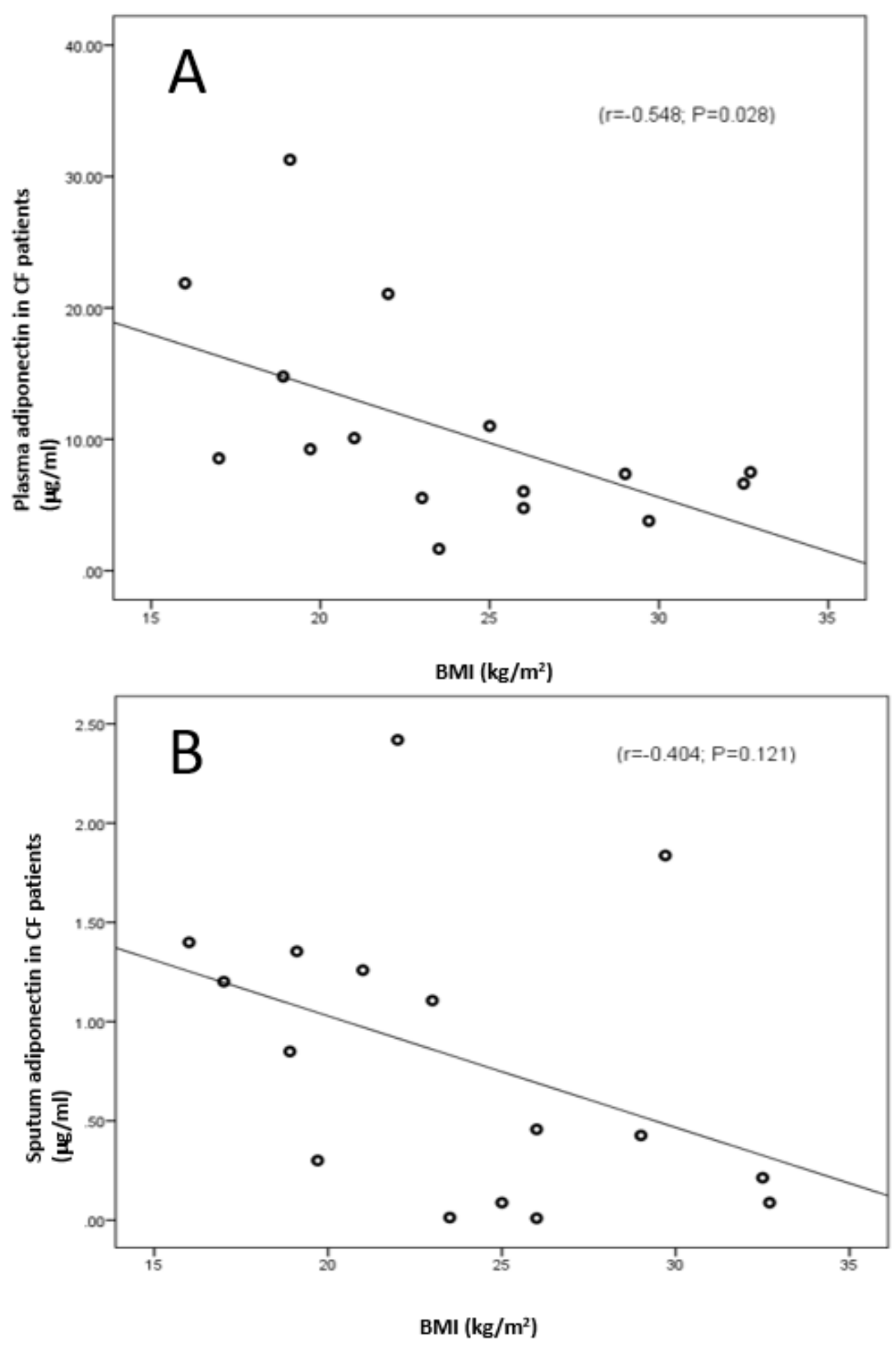

Figure 3

A: Correlation between plasma adiponectin level in $\mathrm{CF}$ patients and BMI. B: Correlation between sputum adiponectin level in CF patients and BMI 